\title{
Insulin resistance and need for a lifestyle change to eliminate it
}

\author{
Mikusova $\mathrm{V}^{1,2}$, Mikus $\mathrm{J}^{2}$, Grilusova $\mathrm{K}^{2}$, Roncakova $\mathrm{M}^{1}$, Benko $\mathrm{J}^{2}$, Martinka $\mathrm{E}^{1}$ \\ National Institute for Endocrinology and Diabetology, Lubochna, Slovakia.vsvakova@gmail.com
}

\begin{abstract}
The AIM of our work is to point out the relationship between insulin resistance and metabolic compensation of diabetes mellitus, as well as to explore the possibilities of improving these parameters by non-drug measures.

The rising incidence of insulin resistance associated with many comorbidities, especially due to the increase in obesity and unhealthy lifestyles, is a serious medical problem today. It is therefore necessary to be able to recognize and evaluate the presence of insulin resistance, prevent its occurrence, and ensure its elimination in high-risk individuals.

In our study, we evaluated 106 patients with diabetes mellitus based on glycated hemoglobin parameters, ratio of triacylglycerols to high-density lipoproteins, and body mass index before and after adjustment of dietary and regime measures.

Statistical analysis of our data showed a positive correlation between the assessed parameters of insulin resistance and metabolic compensation of diabetes with a Pearson correlation coefficient of 0.3156 , and a decrease in glycated hemoglobin and insulin resistance after adjustment of dietary and regimen measures in $73.58 \%$ of patients. Based on the above results, it is shown that non-drug measures are able to significantly improve the parameters of metabolic compensation of diabetes mellitus as well as those of insulin resistance (Tab. 4, Fig. 1, Ref. 18). Text in PDF www.elis.sk

KEY WORDS: insulin resistance, TAG/HDL ratio, diabetes mellitus.
\end{abstract}

\section{Introduction}

Insulin resistance (IR) can be defined as an insufficient biological response to insulin stimulation of target tissues, especially skeletal muscle, adipose and liver tissues, that leads to compensatory hyperinsulinemia. Its metabolic consequences can lead to hyperglycemia, hypertension, dyslipidemia, visceral adiposity, hyperuricemia, increase in inflammatory markers, endothelial dysfunction, prothrombotic condition, and possible progression to metabolic syndrome, atherosclerosis, non-alcoholic steatosis and diabetes mellitus (DM) type 2 (1). IR is thought to precede type $2 \mathrm{DM}$ by 10 to 15 years (2). It is associated not only with obesity, increased cardiovascular risk, prediabetic conditions and DM itself, but also with polycystic ovary syndrome, neuropsychiatric disorders and depression. The prevalence of IR is also rising sharply along with the increasing incidence of obesity and unhealthy lifestyles. Worldwide, the prevalence of IR in adults varies from 15.5 to $46.5 \%$ (3).

Physiologically, it is present in puberty and during pregnancy, and it occurs as well as a transient adaptation phenomenon during

${ }^{1}$ National Institute for Endocrinology and Diabetology, Lubochna, Slovakia, and ${ }^{2}$ Ist Department of Internal Medicine, UNM, Martin, Slovakia

Address for correspondence: V. Mikusova, MD, National Institute for Endocrinology and Diabetology, Lubochna, n.o., Kollarova 282/3, SK-034 91 Lubochna, Slovakia.

Phone: +421907705778 stress response, trauma, and septic infections. In the vast majority of people, IR is caused by acquired risk factors, including nutritional imbalance with weight gain and excess of adipose tissue, insufficient physical activity, aging, drugs (glucocorticoids, antiadrenergics, protease inhibitors, atypical antipsychotics and some exogenous insulins), increased sodium intake, glucose toxicity and lipotoxicity from the excess of circulating free fatty acids. We also know genetic syndromes at risk of IR such as myotonic dystrophy, Alstom's syndrome, Donahue's syndrome, ataxia-telangiectasia, Rabson-Mendenhall's syndrome, Werner's syndrome (4).

In this article, we want to point out the diagnostic possibilities of evaluating IR, especially by use of the ratio of triacylglycerols (TAG) to high-density lipoproteins (HDL). We compare the use of this relatively simple and effective index of IR diagnosis in studies carried out so far. In a group of selected diabetic patients with confirmed unsatisfactory metabolic compensation, we monitor changes in IR and glycated haemoglobin (HbAlc) after adjusting the dietary and regimen measures. Our goal is to point out the importance of following the dietary measures so as to reduce IR and thereby adjust the metabolic compensation.

\section{Material and methods}

In our study, we examined 106 Caucasian patients with DM hospitalized at the National Institute of Endocrinology and Diabetology (NEDU) in the past 5 years, aged 31 to 81 years, mean age 62.32 years ( statistical deviation $(\mathrm{SD})=10.12)$, with DM duration 
Tab. 1. Study group characteristics.

\begin{tabular}{|c|c|c|c|c|c|}
\hline Patients (No. 106) & Male & Female & DM type 1 & DM type 2 & Specific type DM \\
\hline Number (\%) & $73 / 106(68.87 \%)$ & $33 / 106(31.13 \%)$ & $3 / 106(2.83 \%)$ & $101 / 106(95.28 \%)$ & $2 / 106(1.89 \%)$ \\
\hline
\end{tabular}

from 2 to 36 years, average of 18.88 years $(\mathrm{SD}=7.26)$ and other characteristics described in Table 1. Since IR leads to compensatory hyperinsulinemia with subsequent deepening of IR, gradual depletion of pancreatic B-cells with the inability to adequately replace insulin demand, and thus to hyperglycemia (1), we investigated the change in the TAG/HDL ratio as an index for the diagnosis of IR in connection with the change in glycated hemoglobin (HbA1c) as a parameter reflecting DM metabolic compensation.

The oxidation of glucose and fatty acids in the so-called Randle cycle during an increased supply of unsaturated fatty acids (UFA) in muscle tissue results in decreased glucose utilization and thus in IR enhancement. This process is based on a reduction in muscle glycolysis by inhibition of phosphofructokinase due to increased citrate concentrations. Increased concentrations of the fatty acid oxidation products and glucose (acetyl-CoA) inhibit pyruvate dehydrogenase. Similar changes occur in pancreatic B-cells. These are particularly susceptible to the excess of UFA, as their ability to store TAG is very limited, thereby leading to a significant accumulation of toxic lipid metabolites. The condition of hyperglycemia facilitates an increase in production of malonyl-coenzyme A, an inhibitor of carnitine palmitoyl transferase-1 (CPT-1). The subsequent inhibition of CPT-1 impairs the transport of acyl-CoA into the mitochondria, thereby causing the active form of UFA (i.e, with bound coenzyme A) to be used for TAG resynthesis. Long-chain acyl-CoA esters accumulate in the cell and thus cause progressive B-cells dysfunction, which affects insulin production. This takes place in conjunction with the formation of ceramides, representing a strong apoptotic stimulus for B-cells (5).

Although TAG/HDL ratio greater than 3.5 in men and 2.5 in women is considered to be indicative of the presence of IR $(4,6)$, we focused only on patients in whom the TAG/HDL ratio was $\geq$ 3.5 based on lipid profile calculations. We have selected patients according to $\mathrm{HbA} 1 \mathrm{c}$ values, while focusing on diabetics with significantly unsatisfactory metabolic compensation, and determined $\mathrm{HbAlc} \geq 8.5 \%$ as a baseline. Consequently, it was important for us to prove that dietary and regimen measures play an important role in reducing IR and thus adjusting the metabolic compensation of diabetes in these patients. The mentioned repeated education on the basics of regimen and dietary measures included training on carbohydrate units (CU), glycemic index of food, principles of diet of diabetics according to the treatment strategy and type of insulin, and proper self-monitoring of the patient. Therefore, from the group of monitored diabetics, we selected only those in whom there was no change in hypolipidemic treatment or DM treatment. In all of these patients, we performed follow-up examinations of the lipid profile, $\mathrm{HbAlc}$, and BMI after repeated training on proper lifestyle, dietary and regimen measures, and principles of following a proper diabetic low-fat diet during the educational stay. Follow-up examinations were performed on patients after three to twelve months.
In addition to determining the TAG/HDL ratio calculated from the lipid profile, and $\mathrm{HbA} 1 \mathrm{c}$ values, we examined the presence of comorbidities in each patient, especially those related to the presence of IR, dyslipidemia (DLP) and MS such as ischemic heart disease (CHD), ischemic stroke, arterial hypertension (AH), and hepatic steatosis, as well as chronic complications of DM such as diabetic polyneuropathy (DPN), diabetic nephropathy (DN), diabetic retinopathy (DR), cardiac autonomic neuropathy (CAN), peripheral artery disease (PAD), diabetic foot and amputations due to diabetic foot.

The mean baseline BMI in our patients was $33.37(\mathrm{SD}=5.28)$. Of the total number of patients, only 3 subjects $(2.83 \%)$ were not overweight or obese. According to BMI, the overweight was present in $26(24.53 \%)$ patients, grade 1 obesity in 42 (39.62\%) patients, grade 2 obesity in $20(18.87 \%)$ patients, and grade 3 obesity in $15(14.15 \%)$ patients. The duration of the treatment of DM (in form of a combination of diabetic diet with oral antidiabetics in selected patients) was in range of 2-35 years and that of insulin treatment was in range of $2-30$ years. None of the monitored patients was treated solely with the diabetic diet. Out of our study group, 66 patients $(62.26 \%)$ were treated with biguanides, 15 (14.15\%) patients with sulphonylurea derivates, while $9(8.49 \%)$ patients had GLP-1 receptor agonists, 29 (27.36 \%) patients had treatment with DPP-4 inhibitors, and 30 (28.30\%) patients were treated with SGLT-2 inhibitors. None were treated with meglitinide derivatives, alpha-glucosidase inhibitors or thiazolidinediones. Up to $98(92.45 \%)$ patients in the entire cohort were receiving insulin treatment. Of these, there were $69(70.41 \%)$ patients on intensified insulin therapy, $17(17.35 \%)$ patients on conventional insulin therapy, and $12(12.24 \%)$ patients on insulin pumps.

In addition to the low-fat diet, 81 patients $(76.42 \%)$ were treated with hypolipidemic therapy for dyslipidemia. Of the latter 81 patients, 59 patients $(72.84 \%)$ had statins, 58 patients $(71.60 \%)$ had fibrate and $7(8.64 \%)$ were on ezetimibe.

The serum TAG/HDL ratio is a simple index that is effective for the diagnosis of IR; it has been shown to have high sensitivity but low specificity, as it shows different results in different populations depending on ethnicity. Its predictive value was confirmed mainly in Caucasians, while the ratio higher than 3.5 in men or 2.5 in women indicates the presence of IR. $(4,6)$. McLaughlin et al. were the first authors to demonstrate the clinical use of the TAG/ /HDL ratio in identifying overweight individuals (with a BMI above 25) and IR in 258 non-diabetic volunteers (7). According to previous studies, the clinical use of TAG/HDL ratios is also a suitable parameter for assessing metabolic syndrome, DM, atherogenic lipid abnormalities, non-alcoholic hepatic steatosis (NAFLD) and cardiovascular risk $(4,6,8,9)$.

Because serum lipid levels are associated with early atherosclerosis, the use of the TAG/HDL ratio test has been shown to 
Tab. 2. Evaluated parameters.

\begin{tabular}{|c|c|c|c|c|}
\hline & Average before adjustment & SD before adjustment & Average after adjustment & SD after adjustment \\
\hline $\mathrm{HbA1c}$ & 10.46 & 1.38 & 9.55 & 1.45 \\
\hline TAG/HDL & 6.71 & 3.66 & 5.00 & 2.77 \\
\hline $\mathrm{BMI}$ & 33.53 & 5.27 & 33.10 & 5.20 \\
\hline
\end{tabular}

Tab. 3. Statistical evaluation.

\begin{tabular}{lccc}
\hline Correlation & $\mathrm{r}$ & $\mathrm{n}$ & $\mathrm{p}$ \\
\hline HbA1c, TAG/HDL & 0.31562911 & 106 & 0.000981625 \\
TAG/HDL, BMI & 0.063715836 & 106 & 0.516418274 \\
HbA1c, BMI & 0.052982998 & 106 & 0.589607721 \\
\hline
\end{tabular}

be an independent predictor of arterial stiffness in adolescents (10-26 years of age), especially in obese adolescents, which may be useful in the prevention of atherosclerotic diseases (10). In another study with 926 patients, a survey of the prediction of the first cardiovascular event was conducted based on the diagnosis of MS (35\%) and increased TAG/HDL ratio (30\%), again with comparable results. Thus, the increased TAG/HDL ratio appears to be comparable to the diagnosis of MS in predicting the development of cardiovascular disease (CVD) (11). The ability to identify individuals with IR without known heart disease or diabetes by means of TAG/HDL ratio was compared to that of MS parameters, namely measurements of waist circumference, systolic and diastolic blood pressure, fasting plasma insulin, plasma TAG and HDL in 1,102 women and 464 men. The sensitivity and specificity values of MS parameters and TAG/HDL ratio for IR identification were comparable, with $81 \%$ of individuals identically identified (12). Thus, based on these studies, it is clearly reasonable to assume the importance of the TAG/HDL ratio in relation to IR as its predictor, but also to consider it as a useful tool for identifying high-risk individuals so as to prevent or delay the development of IR-related diseases. It is important to remember that the presence of IR is primarily derived from the patient's clinical condition.

\section{Results}

We evaluated $\mathrm{HbA} 1 \mathrm{c}$ as a parameter reflecting the metabolic compensation of diabetes and the TAG/HDL ratio as an index for the diagnosis of IR. We performed a two-tailed paired test for these values before and after lifestyle adjustment and dietary measures with results of 7.41396E-08 for HbA1c and 8.77241E-07 for TAG/ /HDL. Mean values and standard deviation of HbA1c, TAG/HDL and BMI values before and after adjustment of dietary and the regimen measures are shown in Table 2. The parameters in the patient groups before and after adjustment of dietary and regimen measures were subsequently subjected to Pearson correlation analysis. The relationship between BMI difference and $\mathrm{HbA} 1 \mathrm{c}$ difference between the two groups showed an insignificantly positive correlation between BMI reduction and $\mathrm{HbA} 1 \mathrm{c}$ reduction. Similarly, the analysis of the relationship between BMI and TAG/HDL ratio showed that the correlation between the reduction in BMI and reduction in TAG/HDL ratio was insignificantly positive. However, the correlation coefficient in case of the comparison of changes in TAG/HDL and HbA1C was high, thus demonstrating that independently of other factors there was a significant relationship between long-term compensation of diabetes mellitus and insulin resistance. The statistical evaluation is shown in Table 3.

In our study, we observed four variants of the dependence of the ratio of TAG/HDL and HbA1c. The first observed group showed that by changing their lifestyle, especially the eating habits, and following the low-fat diabetic diet, the TAG/HDL ratio as an IR marker decreased over time, while HbA1c decreased as well, thus improving the metabolic compensation of DM. This group of patients represented 78 subjects, i.e, $73.58 \%$ of all patients studied. The second group consisted of patients in whom there was an increase in TAG/HDL and HbA1c, i.e, an increase in glycemia, but also an increase in IR, which again confirmed the theory of using the TAG/HDL ratio as an IR index. In these patients, despite repeated education during hospitalization and outpatient care, there was no desire to make a change in their lifestyle and diet, and their compliance with DM treatment even deteriorated. The second group consisted of $20(18.87 \%)$ patients. The third group consisted of patients whose TAG/HDL ratio decreased but their HbA1c increased. Only three patients out of the total number of diabetics studied ( $2.83 \%$ ) had a given change in the ratio of TAG/HDL to HbA1c. The same was true for the fourth group of patients, who had a decrease in HbA1c but whose TAG/HDL ratio increased. The number of patients in this group was 5 , which is $4.72 \%$ of the total number of patients studied. In these patients, we therefore focused on detailed monitoring of blood glucose levels during hospitalization, i.e, when they were under the strict supervision of medical staff and underwent re-education on the

Dependence of the TAG / HDL ratio and $\mathrm{HbA} 1 \mathrm{c}$

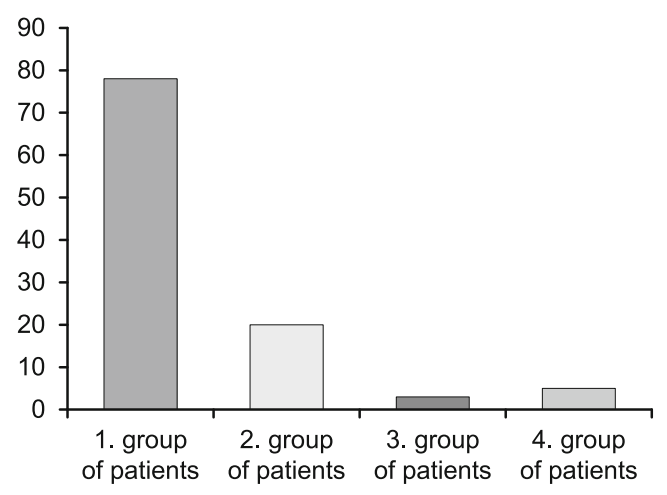

Fig. 1. Dependence of the TAG/HDL ratio and HbA1c. 1) Decrease in both TAG/HDL ratio and HbA1c. 2) Increase in both TAG/HDL ratio and HbA1c. 3) Decrease in TAG/HDL ratio and increase in HbA1c. 4) Increase in TAG/HDL ratio and decrease in HbA1c. 
Tab. 4. Selected comorbidities.

\begin{tabular}{|c|c|c|c|c|c|c|c|c|}
\hline & CHD & Stroke & $\mathrm{AH}$ & $\mathrm{DN}$ & DPN & CAN & PAD & DR \\
\hline Number $(\%)$ & $\begin{array}{c}53 / 106 \\
(50 \%)\end{array}$ & $\begin{array}{c}10 / 106 \\
(9.43 \%)\end{array}$ & $\begin{array}{l}102 / 106 \\
(96.3 \%)\end{array}$ & $\begin{array}{c}77 / 106 \\
(72.64 \%)\end{array}$ & $\begin{array}{c}102 / 106 \\
(96.23 \%)\end{array}$ & $\begin{array}{c}76 / 106 \\
(71.7 \%)\end{array}$ & $\begin{array}{c}42 / 106 \\
(39.62 \%)\end{array}$ & $\begin{array}{c}52 / 106 \\
(49.06 \%)\end{array}$ \\
\hline
\end{tabular}

principles of adherence to proper dietary and lifestyle measures. An illustration of the dependence of the ratio of TAG/HDL and HbA1c in our study can be found in Figure 1.

Along with hyperglycemia and dyslipidemia, smoking is a serious risk factor for the development of atherosclerosis and consequential development of ischemic heart disease (CHD). In our group, smoking was confirmed to be present in case histories of a total of $15(14.15 \%)$ patients. In our study, we also focused on the presence of comorbidities associated with dyslipidemia and $\mathrm{DM}$, such as CHD, stroke, arterial hypertension (AH), and liver disease, which was dominated by hepatic steatosis. These parameters are summarized in Table 4 . Steatofibrosis was confirmed in $24(22.64 \%)$ patients, cirrhosis was diagnosed in $5(4.72 \%)$ patients, one patient suffered from autoimmune hepatitis ( $0.94 \%)$, and $46(43.4 \%)$ patients did not suffer from any liver disease. We also monitored the incidence of endocrinopathies, the largest percentage of which were thyroid diseases. Namely, up to 23 $(21.70 \%)$ patients were treated for thyroid disease and one patient was treated for postoperative hypopituitarism after transnasal resection of pituitary adenoma. Adrenal diseases and other endocrinopathies in the study group were not observed. As to gastrointestinal diseases, 21 patients $(19.81 \%)$ suffered from gastroduodenal ulcer disease, three patients $(2.83 \%)$ had an already developed diabetic gastroparesis, 6 patients $(5.66 \%)$ had chronic gastritis and one patient $(0.94 \%)$ was diagnosed with chronic pancreatitis. A total of $12(11.32 \%)$ patients suffered from cancer. The percentage of chronic organ complications of DM in our group of patients, namely DN, DPN, CAN, DR, and PAD is shown in Table 4. Proteinuria was confirmed from 24-hour urine collection in 40 patients $(37.74 \%)$, one patient $(0.94 \%)$ had already been after kidney transplantation, and none of the monitored patients had currently been in the dialysis program. Diabetic foot syndrome was present in 8 patients $(7.55 \%)$ and there were 5 patients $(4.72 \%)$ already after lower limb amputation, while 52 patients (49.06\%) were diagnosed with retinopathy.

\section{Discussion}

In a study comparing the diagnostic accuracy of TAG/HDL, HOMA-IR, ratio of total cholesterol and HDL to predict MS, IR and dyslipidemia in 1,932 non-diabetic individuals from Germany, the TAG-to-HDL ratio appeared to be the most relevant and practical index for predicting this risk (13). Studies from 2015 and 2017 confirmed that children with significantly higher HOMA-IR values (depending on age, gender and BMI), as well as those with higher insulin levels, acanthosis nigricans and metabolic syndrome, had a higher TAG/HDL ratio. The TAG/HDL ratio correlated with HOMA-IR and waist circumference; therefore, it can be used as a relatively simple biochemical marker for IR $(14,8)$. A 15-year prospective study to demonstrate a predisposition to a higher TAG/ /HDL ratio to DM development confirmed that TAG and TAG/ /HDL ratio were independent risk factors for DM. The incidence of DM increased with an increase in TAG/HDL ratio. The clinical use of TAG and HDL values is therefore justified not only in the identification of patients with IR but also in those with DM (15). Significantly higher TAG values with lower HDL, as compared to the control group, were shown in subjects with IR or DM in a study of 9,764 Chinese participants. The Pearson correlation analysis showed that as compared to other lipid parameters, TAG and HDL lipid values were significantly correlated with fasting insulin, fasting glycemia, glycemia after 2 hours during oGTT, and HbA1c (9). In our study, the decrease in TAG/HDL ratio significantly correlated with the decrease in HbAlc values, thus reflecting the metabolic compensation of diabetes mellitus and decrease in IR. Out of the total of 106 diabetics, HbA1c reduced in up to 78 patients alongside with the decrease in TAG/HDL ratio. The correlation of the relationship of the TAG/HDL ratio as an index for the diagnosis of IR and metabolic compensation of diabetes mellitus with HbA1c was also confirmed in other $18.87 \%$ of patients, in whom both TAG/HDL ratio HbAlc increased. Together, we confirmed the effectiveness of the TAG/HDL ratio in utilizing it as an index of IR diagnosis in up to $92.45 \%$ of patients.

As already mentioned, IR arises most often as an acquired condition caused by incorrect lifestyle with excessive energy intake, and insufficient physical activity, thus leading to obesity as the basic risk factor. In terms of treatment as well as prevention, it is therefore of paramount importance to make an intervention in lifestyle in form of restriction of saturated fats, supplementation of polyunsaturated fatty acids, increase in fiber intake, decrease in $\mathrm{NaCl}$ intake ( $\max 3-6 \mathrm{~g} /$ day), regular physical activity, weight reduction (by 5-7\%), control and adjustment of blood pressure and smoking cessation. Individuals with IR are at high risk of developing type $2 \mathrm{DM}$. In people aged 60 and over, the risk of developing DM was reduced by $70 \%$ through a change in lifestyle (16). Weight loss of $7 \%$ reduced the onset of type $2 \mathrm{DM}$ in individuals with IR by up to $58 \%$, whereas in the other arm of individuals with IR, the risk of DM was reduced by only $31 \%$ after the addition of metformin to treatment (4). In our monitored patients, we saw that after thorough and repeated education of patients, the TAG/ /HDL ratio decreased as an index for the diagnosis of IR and thus improved metabolic compensation of diabetes mellitus by decreasing $\mathrm{HbA} 1 \mathrm{c}$ values in up to $73.58 \%$ of patients. It is also important to note that the group of diabetics we monitored included not only patients with type $2 \mathrm{DM}$ but also patients with type $1 \mathrm{DM}$, as well as patients with a specific type of DM. It is therefore essential to think about the development of IR in connection with unhealthy lifestyle choices and non-compliance with the dietary and regimen measures in type 1 diabetics. 
The TAG/HDL ratio has been shown to be a useful indicator of atherogenic lipid abnormalities, IR and cardiovascular disease. In a sample of children and adolescents with normal BMI and those overweight to obese, the relationship of the TAG/HDL ratio with early signs of morphological vascular changes and cardiometabolic risk factors, including non-alcoholic hepatic steatosis (NAFLD), was assessed. The study showed that the TAG/HDL ratio increased along with the incidence of central obesity, IR, elevated hsCRP, NAFLD, MS, intimal-medial carotid thickness (cIMT) (increased significantly with age), gender and BMI, which may help to identify children and adolescents at high risk of structural, vascular and metabolic changes (17). In an analysis of 39,447 men, the TAG/ /HDL ratio was monitored in the prediction of type $2 \mathrm{DM}$, coronary heart disease, and CVD. The incidence of DM was twice as high in subjects with a high TAG/HDL ratio as compared to those with low TAG/HDL ratio, and the mortality from CVD was also higher in subjects, in whom the TAG/HDL ratio was higher. The TyG index was also monitored, which predicted the incidence of DM but not the mortality from cardiovascular disease (18). In our study, we found that 53 patients $(50 \%)$ out of the total number of diabetics with confirmed IR by means of using TAG/HDL ratio had the diagnosis of coronary heart disease and $9.43 \%$ had a stroke. As many as 102 patients $(96.3 \%)$ were diagnosed with arterial hypertension and $39.62 \%$ suffered from PAD.

\section{Conclusion}

In our thesis, we wanted to point out the possibility of using the TAG/HDL ratio as a simple and effective index in determining the presence of IR in our population. As IR is primarily associated with poor lifestyle choices and rising incidence of obesity, the interference with regimen and dietary measures is fundamental to the elimination of IR. The relationship between the TAG/HDL ratio and $\mathrm{HbA} 1 \mathrm{c}$ was proved to be highly significant, whereas the correlation between $\mathrm{BMI}$ reduction and $\mathrm{HbA} 1 \mathrm{c}$ reduction and the relationship between BMI and TAG/HDL ratio were not significant. It is also important to remember that IR may be present not only in patients with type $2 \mathrm{DM}$, as it can develop also in patients with type $1 \mathrm{DM}$ due to unhealthy lifestyle choices and failure to follow proper regimens along with the diabetic diet.

\section{References}

1. Deacon CF. Physiology and Pharmacology of DPP-4 in Glucose Homeostasis and the Treatment of Type 2 Diabetes. Front Endocrinol 2019; 10 (80): 1-14.

2. Hossan T, Kundu S et al. Epigenetic Modifications Associated with the Pathogenesis of Type 2 Diabetes Mellitus. Endocrine Metab Immune Dis Drug Targets 2019; 19 (6): 775-786.
3. Fahed M, Jaoudeh MG et al. Evaluation of risk factors for insulin resistance: a cross sectional study among employees at a private university in Lebanon. BMC Endocrine Dis 2020; 20: 85.

4. Freeman AM, Pennings N. Insulin Resistance. Stat Pearls 2020. https: //www.ncbi.nlm.nih.gov/books/NBK507839/.

5. Kusminski CM, Shetty S et al. Diabetes and apoptosis: lipotoxicity. Apoptosis 2009; 14 (12): 1484-1495.

6. Mazidi M, Kengne AP et al. Lipid accumulation product and triglycerides/glucose index are useful predictors of insulin resistance. J Diabet Complicat 2018; 32( 3): 266-270.

7. McLaughlin T, Abbasi F et al. Use of metabolic markers to identify overweight individuals who are insulin resistant. Ann Intern Med 2003; 139 (10): 802-809.

8. Iwani AKZ, Jalaludin MY et al. Triglyceride to HDL-C Ratio is Associated with Insulin Resistance in Overweight and Obese Children. Scie Reports 2017; 7 (40055): 1-7.

9. Lin D, Qi Y et al. Associations of lipid parameters with insulin resistance and diabetes: A population-based study. Clin Nutrition 2018; 37 (4): 1423-1429.

10. Urbina EM, Khoury PR et al. Triglyceride to HDL-C Ratio and Increased Arterial Stiffness in Children, Adolescents, and Young Adults. Pediatrics 2013; 131 (4): 1082-1090.

11. Salazar MR, Carbajal HA et al. Identifying cardiovascular disease risk and outcome: use of the plasma triglyceride/high-density lipoprotein cholesterol concentration ratio versus metabolic syndrome criteria. J Intern Med 2013; 273 (6): 595-601.

12. Salazar MR, Carbajal H. et al. Comparison of the abilities of the plasma triglyceride/high-density lipoprotein cholesterol ratio and the metabolic syndrome to identify insulin resistance. Diabet Vasc Dis Res 2013; 10 (4): 346-352.

13. von Bibra $\mathbf{H}$, Saha $\mathbf{S}$ et al. Impact of the triglyceride/high-density lipoprotein cholesterol ratio and the hypertriglyceremic-waist phenotype to predict the metabolic syndrome and insulin resistance. Hormone and metabolic research $=$ Hormon und Stoffwechselforschung $=$ Hormones et metabolisme 2017; 49 (7): 542-549.

14. Hirschler V, Maccallini G et al. Association between triglyceride to HDL-C ratio and insulin resistance in indigenous Argentinean children. Pediat Diabet 2015; 16 (8): 606-612.

15. He S, Wang S et al. Higher ratio of triglyceride to high-density lipoprotein cholesterol may predispose to diabetes mellitus: 15-year prospective study in a general population. Metabolism 2012; 61 (1): 30-36.

16. Gazdíková K. Prediabetes. Via practica 2017; 14 (1): 16-20.

17. Pacifico L, Bonci E et al. Association of serum triglyceride-to-HDL cholesterol ratio with carotid artery intima-media thickness, insulin resistance and nonalcoholic fatty liver disease in children and adolescents. Nutrit Metab Cardiovasc Dis 2014; 24 (7): 737-743.

18. Vega GL, Barlow CE et al. Triglyceride-to-high-density-lipoproteincholesterol ratio is an index of heart disease mortality and of incidence of type 2 diabetes mellitus in men. J Invest Med 2014; 62 (2): 345-349.

Received December 28, 2020. Accepted January 24, 2021. 